\title{
Elucidating the Nature of the Fine Structure Constant and Indicating the Existence of an Unknown Angular Momentum
}

\author{
Koshun Suto ${ }^{1}$ \\ ${ }^{1}$ Chudaiji Buddhist Temple, Isesaki, Japan \\ Correspondence: Koshun Suto, Chudaiji Buddhist Temple, Isesaki, Japan. Tel: 81-270-23-9980. E-mail: \\ koshun_suto129@mbr.nifty.com
}

Received: July 8, 2017

Accepted: July 18, 2017

Online Published: July 24, 2017

doi:10.5539/apr.v9n4p17

URL: https://doi.org/10.5539/apr.v9n4p17

\begin{abstract}
In this paper, the author searches for a formula different from the existing formula in order to elucidate the nature of the fine structure constant $\alpha$. The relativistic energy of the electron in a hydrogen atom is expressed as $E_{\mathrm{re}, n}$ and the momentum corresponding to that energy is taken to be $p_{\mathrm{re}, n}$. Also, $p_{\mathrm{p}, n}$ is assumed to be the momentum of a photon emitted when an electron that has been stationary in free space transitions to the inside of a hydrogen atom. When $n=1$, the ratio of $p_{\mathrm{re}, 1}$ and $p_{\mathrm{p}, 1}$ matches with $\alpha$. That is, $p_{\mathrm{p}, 1} / p_{\mathrm{re}, 1}=\alpha$. Also, the formula for the energy of a photon is $E=h v$. However, this formula has no constant of proportionality. If one wishes to claim that the energy of a photon varies in proportion to the photon's frequency, then a formula containing a constant of proportionality is necessary. Thus, this paper predicts that, in the natural world, there is a minimum unit of angular momentum $h_{\mathrm{vp}}$ smaller than the Planck constant. (The vp in $h_{\mathrm{vp}}$ stands for "virtual particle.")

If this physical constant is introduced, then the formula for the energy of the photon can be written as $E=h_{v p} v / \alpha$. If $h_{\mathrm{vp}}$ exists, a formula can also be obtained which helps to elucidate the nature of the fine structure constant.
\end{abstract}

Keywords: Fine structure constant, Relativistic energy, Hydrogen atom, Planck constant

\section{Introduction}

The fine structure constant $\alpha$ is a mysterious constant which has no dimensions, and its value is defined as follows.

$$
\alpha=\frac{e^{2}}{4 \pi \varepsilon_{0} \hbar c} .
$$

This constant was introduced in 1916 by Sommerfeld to explain the slight splitting (fine structure) of spectral lines for the hydrogen atom. However, its nature is not well understood. Therefore, this paper searches for a formula different from Equation (1), and thereby aims to deepen understanding of the fine structure constant.

This paper also searches for an unknown physical constant with the dimensions of angular momentum. The reason is dissatisfaction with the following formula for the energy of a photon.

$$
E=h v \text {. }
$$

The energy of a photon matches the product of the Planck constant $h$ and the photon's frequency $v$. However, in general, the tendency is to regard $E$ as varying in proportion with $v$. In that case, the Planck constant is regarded as the constant of proportionality. However, $h$ has dimensions, and thus this constant cannot be regarded as a constant of proportionality. It may be possible to solve this problem if an unknown physical constant exists which has the dimensions of angular momentum. In that case, first the dimensions of energy are obtained from the product of the unknown angular momentum and $v$. Next, the constant of proportionality is multiplied with that product, and the numerical value is made to match with energy.

If that sort of formula can be obtained, then it will also be possible to clarify the relationship between the constant of proportionality contained in the unknown formula and the fine structure constant.

\section{A Formula Applicable to the Electron in a Hydrogen Atom}

Letting $m_{0} c^{2}$ be the rest mass energy and $\boldsymbol{p}$ the momentum of an object or a particle existing in free space, Einstein's energy-momentum relationship is given by the following equation: 


$$
\left(m c^{2}\right)^{2}=\boldsymbol{p}^{2} c^{2}+\left(m_{0} c^{2}\right)^{2} .
$$

Here, $m c^{2}$ is the relativistic energy.

In contrast, the author has derived the following relationship for a bound electron in a hydrogen atom, which must take into account the Coulomb potential (Suto, 2011):

$$
E_{\mathrm{re}, n}^{2}+\boldsymbol{p}_{n}^{2} c^{2}=\left(m_{\mathrm{e}} c^{2}\right)^{2}, \quad n=1,2, \cdots
$$

Here, $E_{\mathrm{re}, n}$ is the relativistic energy described on an absolute scale. $E_{\mathrm{re}, n}$ expresses the remaining amount of rest mass energy of the electron.

Incidentally, Bohr derived the following formula for the energy levels in the hydrogen atom. This formula can be obtained from the sum of potential energy and kinetic energy. (Naturally, application of the quantum condition is indispensable).

$$
E_{n}=V\left(r_{n}\right)+K_{n}=\frac{1}{2} V\left(r_{n}\right)=-K_{n}=-\frac{1}{2}\left(\frac{1}{4 \pi \varepsilon_{0}}\right)^{2} \frac{m_{\mathrm{e}} e^{4}}{\hbar^{2}} \cdot \frac{1}{n^{2}}, \quad n=1,2, \cdots
$$

$E_{n}$ in Equation (5) is total mechanical energy. However, in itself $E_{n}$ is an energy which should be defined as follows.

$$
E_{n}=E_{\mathrm{re}, n}-m_{\mathrm{e}} c^{2}
$$

$E_{n}$ expresses the reduction in rest mass energy of the electron. Also $E_{\mathrm{re}, n}$ can be defined as follows.

$$
E_{\mathrm{re}, n}=m_{\mathrm{re}, n} c^{2} \text {. }
$$

Here, $m_{\mathrm{re}, n}$ is the relativistic mass of the electron.

Incidentally, it is known that the following formula can be derived from Equation (1).

$$
E=m_{0} c^{2}\left(1-\frac{v^{2}}{c^{2}}\right)^{-1 / 2} \text {. }
$$

If the same logic is applied to Equation (4), then the following formula can be derived.

$$
E_{\mathrm{re}}=m_{\mathrm{e}} c^{2}\left(1+\frac{v^{2}}{c^{2}}\right)^{-1 / 2}
$$

Here, the following assumption is made (Suto, 2017):

$$
\frac{v_{n}}{c}=\frac{\alpha}{n}, \quad n=1,2, \cdots
$$

However, this velocity is taken to be the average velocity of the electron, in accordance with quantum mechanics. The following formula for $E_{\mathrm{re}, n}$ is obtained from Equations (9) and (10).

$$
E_{\mathrm{re}, n}=m_{\mathrm{e}} c^{2}\left(\frac{n^{2}}{n^{2}+\alpha^{2}}\right)^{1 / 2}, \quad n=1,2, \cdots
$$

From this, Equation (4) can be written as follows.

$$
\left[m_{\mathrm{e}} c^{2}\left(\frac{n^{2}}{n^{2}+\alpha^{2}}\right)^{1 / 2}\right]^{2}+\left[m_{\mathrm{e}} c^{2}\left(\frac{\alpha^{2}}{n^{2}+\alpha^{2}}\right)^{1 / 2}\right]^{2}=\left(m_{\mathrm{e}} c^{2}\right)^{2} . \quad n=1,2, \cdots
$$

Also, if Equation (12) is rewritten into a relation of momentum, the result is as follows.

$$
\left[m_{\mathrm{e}} c\left(\frac{n^{2}}{n^{2}+\alpha^{2}}\right)^{1 / 2}\right]^{2}+\left[m_{\mathrm{e}} c\left(\frac{\alpha^{2}}{n^{2}+\alpha^{2}}\right)^{1 / 2}\right]^{2}=\left(m_{\mathrm{e}} c\right)^{2} .
$$

The steps thus far reconfirm matters already discussed in other papers (Suto, 2017): 
The two terms on the left side of Equation (13) can be regarded as vectors, and the magnitude of these vectors is defined as follows.

$$
\begin{aligned}
& p_{\mathrm{re}, n}=m_{\mathrm{e}} c\left(\frac{n^{2}}{n^{2}+\alpha^{2}}\right)^{1 / 2} . \\
& p_{\mathrm{p}, n}=m_{\mathrm{e}} c\left(\frac{\alpha^{2}}{n^{2}+\alpha^{2}}\right)^{1 / 2} .
\end{aligned}
$$

$p_{\mathrm{re}, n}$ in Equation (14) is the momentum of the electron corresponding to the relativistic energy $m_{\mathrm{re}, n} c^{2}$. Now, what is $p_{\mathrm{p}, n}$ in Equation (15)?

The following relationship holds for energy of an electron in a hydrogen atom.

$$
m_{\mathrm{e}} \mathrm{c}^{2}=E_{\mathrm{re}, n}-E_{n}=E_{\mathrm{re}, n}-\left(K_{n}+V\left(r_{n}\right)\right)=\left(m_{\mathrm{e}} c^{2}-K_{n}\right)-E_{n}=\left(m_{\mathrm{e}} c^{2}-K_{n}\right)+\hbar \omega, \quad E_{n}<0 .
$$

Here, $\hbar \omega$ is the energy of a photon emitted when an electron that has been stationary in free space is taken into the hydrogen atom.

According to Equation (16), the kinetic energy of the electron is included in $E_{\mathrm{re}, n}$, and thus the momentum of the electron is also naturally included in $p_{\mathrm{re}, n}$ in Equation (14). Therefore, in this paper, it is predicted that $p_{\mathrm{p}, n}$ is the momentum of a photon emitted when an electron that has been stationary in free space transitions to the inside of a hydrogen atom. (The $\mathrm{p}$ in $p_{\mathrm{p}, n}$ stands for photon.)

It is evident from Equation (13) that the two momentum vectors are orthogonal. Also, Equation (13) can be written as follows.

$$
p_{\mathrm{re}, n}^{2}+p_{\mathrm{p}, n}^{2}=\left(m_{\mathrm{re}, n} c\right)^{2}+\left(\frac{\alpha}{n} m_{\mathrm{re}, n} c\right)^{2}=\left(m_{\mathrm{e}} c\right)^{2}, \quad m_{\mathrm{re}, n}=m_{\mathrm{e}}\left(1+\frac{\alpha^{2}}{n^{2}}\right)^{-1 / 2} .
$$

From this, the following relation can be derived.

$$
\frac{p_{\mathrm{p}, n}}{p_{\mathrm{re}, n}}=\frac{\alpha}{n}, \quad n=1,2, \cdots
$$

When $n=1$, Equation (18) matches with the fine structure constant.

\section{Region where Virtual Particle Pairs Constituting the Vacuum can Exist}

The content of this section is treated in multiple papers, but is regarded as necessary for the discussion in Section 4 (Suto, 2014, 2017):

The author presented the following equation as an equation indicating the relationship between the rest mass energy and potential energy of the electron (Suto, 2009):

$$
V(r)=-\Delta m_{\mathrm{e}} c^{2}
$$

According to this equation, the potential energy of a bound electron in a hydrogen atom is equal to the reduction in rest mass energy of that electron.

There is a lower limit to potential energy, and the range which energy can assume is as follows.

$$
-m_{\mathrm{e}} c^{2} \leq V(r)<0 .
$$

Also, the following constraint holds regarding the relativistic energy $E_{\text {re }}$ of the electron due to Equations (5) and (6) (here, the discussion is limited to the ordinary energy levels of the atom).

$$
\frac{1}{2} m_{\mathrm{e}} c^{2} \leq E_{\mathrm{re}}<m_{\mathrm{e}} c^{2}
$$

The logic used when deriving Equation (2) can also be applied in the region of Equation (21).

Incidentally, the energy of the electron in a hydrogen atom can be given not only by Equation (11) but also by the following formula.

$$
E=E_{\mathrm{re}}-m_{\mathrm{e}} c^{2}=\frac{1}{2} V(r)=-\frac{1}{2} \frac{e^{2}}{4 \pi \varepsilon_{0} r} .
$$


Here, if $-m_{\mathrm{e}} c^{2}$ is substituted for $E$ in Equation (22), then the $r$ where $E_{\mathrm{re}}=0$ is

$$
r=\frac{r_{\mathrm{e}}}{2} .
$$

Here, $r_{\mathrm{e}}$ is the classical electron radius as follows.

$$
r_{\mathrm{e}}=\frac{e^{2}}{4 \pi \varepsilon_{0} m_{\mathrm{e}} c^{2}} .
$$

Also, according to Equation (22), the following relationship holds between $E_{\mathrm{re}}$ and $r$.

$$
E_{\mathrm{re}}=\frac{m_{\mathrm{e}} c^{2}}{2} \leftrightarrow r=r_{\mathrm{e}} .
$$

Incidentally, there are positive and negative solutions for the energy in Equation (4) (Suto, 2014):. Here, the energy levels of an ordinary hydrogen atom are expressed as $E_{\mathrm{re}, n}^{+}$and the unknown energy levels are expressed as $E_{\mathrm{re}, n}^{-}$ At this time, the following relations hold between $r$ and $E$.

$$
\begin{aligned}
r_{\mathrm{e}}<r_{n}^{+} & \leftrightarrow \frac{1}{2} m_{\mathrm{e}} c^{2}<E_{\mathrm{re}, n}^{+}<m_{\mathrm{e}} c^{2} . \\
\frac{r_{\mathrm{e}}<r_{n}^{-}<\frac{r_{\mathrm{e}}}{3}}{4} & \leftrightarrow-m_{\mathrm{e}} c^{2}<E_{\mathrm{re}, n}^{-}<-\frac{1}{2} m_{\mathrm{e}} c^{2} . \\
r & =\frac{r_{\mathrm{e}}}{2} \leftrightarrow E_{\mathrm{re}}=0 .
\end{aligned}
$$

As is clear from Equations. (26.1) and (26.2), the electron cannot penetrate into the following region.

$$
\frac{r_{\mathrm{e}}}{3} \leq r \leq r_{\mathrm{e}} \leftrightarrow-\frac{1}{2} m_{\mathrm{e}} c^{2} \leq E_{\mathrm{re}} \leq \frac{1}{2} m_{\mathrm{e}} c^{2} .
$$

Equation (4) is applicable in the ranges of Equations (26.1) and (26.2). Therefore, in this paper, the region in Equation (27) is predicted to be a region where there exist virtual electron and positron pairs constituting the vacuum. However, the energy of a virtual particle pair is twice the energy of the virtual electron, and thus Equation (27) can be rewritten as follows.

$$
\frac{r_{\mathrm{e}}}{3} \leq r_{\mathrm{vp}} \leq r_{\mathrm{e}} \leftrightarrow-m_{\mathrm{e}} c^{2} \leq E_{\mathrm{vp}} \leq m_{\mathrm{e}} c^{2} .
$$

Here, $E_{\mathrm{vp}}$ indicates the energy of virtual particle pairs (the subscript "vp" stands for "virtual particle.") A subscript is also attached to $r$ to take symmetry into account.

If a classical picture is used, then $r_{\mathrm{e}}$ is not the radius of virtual particle pairs in orbital motion, and can be regarded as the distance from the center of atomic nucleus to the virtual particle pair.

Incidentally, potential energy does not exist in the region of Equation (27). Therefore, if it is assumed that the energy-momentum relationship holds for virtual particle pairs too, then that can be obtained by setting $m_{0}$ equal to zero in Equation (3). That is,

$$
E_{\mathrm{vp}}=m_{\mathrm{vp}} c^{2}=p_{\mathrm{vp}} c \text {. }
$$

Here, $m_{\mathrm{vp}}$ indicates the mass of the virtual particle pair, and $p_{\mathrm{vp}}$ indicates the momentum of the virtual particle pair.

\section{Introduction of Unknown Angular Momentum and Formula for the Energy of a Photon}

Bohr assumed the following quantum condition when constructing the classical quantum theory.

$$
p_{n} \cdot 2 \pi r_{n}=2 \pi n \hbar, \quad n=1,2, \cdots .
$$

Following that approach, this paper creates a physical quantity with the dimensions of angular momentum from the virtual particle pair. If Equations (28) and (29) are used, then $p_{\mathrm{vp}}$ and $r_{\mathrm{vp}}$ become as follows when the energy of the virtual particle pairs is $m_{\mathrm{e}} c^{2}$.

$$
p_{\mathrm{vp}}=m_{\mathrm{e}} \mathrm{c}, \quad r_{\mathrm{vp}}=r_{\mathrm{e}} .
$$

From this, the following units of angular momentum can be created. 


$$
p_{\mathrm{vp}} r_{\mathrm{vp}}=\frac{e^{2}}{4 \pi \varepsilon_{0} c}=\alpha \hbar .
$$

Here, this paper defines the following quantity with the dimensions of angular momentum.

$$
\begin{gathered}
\hbar_{\mathrm{vp}}=\frac{h_{\mathrm{vp}}}{2 \pi}=\frac{e^{2}}{4 \pi \varepsilon_{0} c} . \\
h_{\mathrm{vp}}=\frac{e^{2}}{2 \varepsilon_{0} c}=4.835276921 \times 10^{-36} \mathrm{Js} .
\end{gathered}
$$

$h_{\mathrm{vp}}$ can be regarded as the intrinsic angular momentum for a virtual particle pair. In response, the Planck constant can be written as follows.

$$
h=m_{\mathrm{e}} c \lambda_{\mathrm{C}} .
$$

The Planck constant is the intrinsic angular momentum for an electron. Also, from Equation (33), the relationship between $\hbar$ and $\hbar_{\mathrm{vp}}$ becomes as follows.

$$
\hbar_{\mathrm{vp}}=\alpha \hbar
$$

Here, if the ratio of $h$ and $h_{\mathrm{vp}}$ is taken,

$$
\frac{\hbar \mathrm{vp}}{\hbar}=\frac{h_{\mathrm{vp}}}{h}=\alpha .
$$

The fine structure constant can be defined as the ratio of $h$ and $h_{\mathrm{vp}}$. If the relationship in Equation (37) is used, then Equation (2) can be written as follows.

$$
E=\frac{1}{\alpha} h_{\mathrm{vp}} v .
$$

The energy of a photon varies in proportion with $h_{\mathrm{vp}} v$ (in this case, $1 / \alpha$ is the constant of proportionality). If the formula for the energy of a hydrogen atom is expressed as in Equation (38), this resolves the dissatisfaction of the author which was raised a problem in the introduction.

When deriving Equation (5), Bohr assumed Equation (30). If discrete energy levels also exist in the virtual particle pairs constituting the vacuum, then a formula is necessary to describe that. In that case, a good approach is to assume the following quantum condition, and then derive the energy levels of the vacuum (Suto, 2015):

$$
2 \pi p_{\mathrm{vp}, n} r_{\mathrm{vp}, n}=2 \pi n \alpha \hbar \text {. }
$$

\section{Conclusion}

1) If $n=1$ is set in Equation (18), then the fine structure constant can be defined as follows.

$$
\frac{p_{\mathrm{p}, 1}}{p_{\mathrm{re}, 1}}=\alpha .
$$

Also, if the relation in Equation (39) holds for virtual particle pairs constituting the vacuum, then the following relation can be obtained from Equations (30) and (39).

$$
\frac{p_{\mathrm{vp}, n} r_{\mathrm{vp}, n}}{p_{n} r_{n}}=\alpha, \quad n=1,2, \cdots
$$

2) In the natural world, there is a unit of angular momentum smaller than the Planck constant. That is,

$$
h_{\mathrm{vp}}=\frac{e^{2}}{2 \varepsilon_{0} c}=4.835276921 \times 10^{-36} \mathrm{Js} .
$$

It can be predicted that this physical constant is the intrinsic angular momentum for a virtual particle pair. The ratio of $h_{\mathrm{vp}}$ and $h$ is as follows.

$$
\frac{h_{\mathrm{vp}}}{h}=\alpha .
$$

If this unknown physical constant $h_{\mathrm{vp}}$ is introduced into physics, then Equation (2) can be written as follows. 


$$
E=\frac{1}{\alpha} h_{\mathrm{vp}} v .
$$

The author is convinced that the discussion in this paper has yielded new findings regarding the fine structure constant.

\section{Acknowledgements}

I would like to express my thanks to the staff at ACN Translation Services for their translation assistance.

\section{References}

Suto, K. (2009). True nature of potential energy of a hydrogen atom. Physics Essays, 22(2), 135-139. http://dx.doi.org/10.4006/1.3092779

Suto, K. (2011). An energy-momentum relationship for a bound electron inside a hydrogen atom. Physics Essays, 24(2), 301-307. http://dx.doi.org/10.4006/1.3583810

Suto, K. (2014). Previously unknown ultra-low energy level of the hydrogen atom whose existence can be predicted. Applied Physics Research, 6(6), 64-73. http://dx.doi.org/10.5539/apr.v6n6p64

Suto, K. (2015). An unknown physical constant missing from physics. Applied Physics Research, 7(5), 68-79. http://dx.doi.org/10.5539/apr.v7n5p68

Suto, K. (2017). Previously unknown physical formulas which hold in a hydrogen atom and are derived without using quantum mechanics. Applied Physics Research, 9(3), 7-16. https://doi.org/10.5539/apr.v9n3p7

\section{Copyrights}

Copyright for this article is retained by the author(s), with first publication rights granted to the journal.

This is an open-access article distributed under the terms and conditions of the Creative Commons Attribution license (http://creativecommons.org/licenses/by/4.0/). 